\title{
Spoonful Dosing Unit
}

National Cancer Institute

\section{Source}

National Cancer Institute. Spoonful Dosing Unit. NCI Thesaurus. Code C149942.

A unit of presentation used to represent the quantity of product that is administered by filling a single spoon administration device. 